\title{
The Nature of CVC Nonlinearity in Low-Voltage Scanning Tunneling Spectroscopy of Semiconductors
}

\author{
A.I. Loskutov*, A.M. Mandel, E.E. Karpova, V.B. Oshurko, S.G. Veselko, A.A. Sharts and S.A. Loskutov \\ *Moscow State Technological University “STANKIN”, RU-127055, Moscow, Russian Federation;
}

\begin{abstract}
A new model of field emission in a scanning tunnelling microscope was developed. The model describes the tunnelling current from a surface of semiconductor (semimetal) and allows estimating the preexponential factor in the expression for the tunneling probability. It is shown that this factor is directly related to the degree of localization of the electron density and determines the shape of the local tunnel current-voltage characteristics (LTCVCs) at low voltages. The model allows separating the contributions of surface electronic states of different symmetry (dimension) of the tunnelling current. The practical application of the model is demonstrated by the example of mathematical processing of the LTCVCs of HOPG surface containing different structural defects.

Keywords: charge tunneling, STM, STS, CVC, surface defects, monographite.
\end{abstract}

\section{Introduction}

The processes of assembling two-dimensional monolayers of organic molecules constantly attract the attention of researchers. They are observed in both liquid and solid surfaces. However, for their practical applications, such as functional elements of organic nano and microelectronics one must use substrate surface acts as a template for self-assemble two-dimensional monolayers and determines their final structure and electrical properties.

It is well known that the defects on the surface of solids are active sites in catalysis, adsorption and nucleating centers of a new phase [1-3], because it is on them free electrons are localized [4]. For example, randomly distributed charges, which are presented in the silicon oxide layer causes large potential fluctuations and substantially affect the structure of graphene deposited on the silicon surface [5].

There are different ways of visualizing structural defects on the surface of solids: by destructive mode (chemical, electrochemical, ion-plasma etching) and nondestructive mode (selective adsorption, electron and probe microscopies). However, local analysis of their electronic properties and their distribution over the surface are a more complicated problem.

The main objective of this work is experimentally test a new methodology for analyzing the LTCVCs, which allows one to separate the contributions of different electronic states to the tunneling current. This will enable separating "normal" surface electrons delocalized in the plane of the sample surface, from electrons, localized at surface defects, and thereby estimate the local density and type of the latter. This study is not only of theoretical interest, but also has practical value. Especially it is necessary for monitoring semiconductors surface properties in micro and nanoelectronic technologies, because defects disturb lateral mobility of the electrons.

At low electric fields electron emission from the semiconductor surface, when is measured by probe microscopy techniques, is a particular case of the tunnelling effect [6]. Such processes are usually studied with the semi-classical approximation that determines the amplitude of the transition up to an exponential factor.

In this voltage range electric field only slightly reduces the average height of the potential barrier at solid surface and its strength disappears from the exponent of the estimated CVC. In the same time, the pre-exponential factor in the transition amplitude is not taken into account at all. Since the density of electronic states, participating in the transition and "liberated" by the electric field on the surface of the probe, is proportional to the voltage $U$, the theoretical CVC of the tunnel current usually is purely ohmic at low voltages: $\mathrm{I} \sim \mathrm{U}$.

At the same time, numerous experiments show that the real CVC deviates from the linear law even in the vicinity of $U=0$. In this article we discuss the possible physical nature of this deviation and demonstrate that taking into account the above-mentioned pre-exponential factor it is possible to explain the tunneling probability and physically interpret such deviations. Unfortunately, the exact solution of the threedimensional Schrodinger equation, describing electron tunneling from the real surface (i.e. containing various types of defects) of the semiconductor, is impossible for various reasons. Practically at low fields, it has no influence on the exponential factor of the tunnelling probability, but it is critically important for the pre-

* Corresponding author: ailoskutov@yandex.ru, tel.+79164919418 
exponential factor, which determines the transition probability and, therefore, forms the tunneling current. To solve the problem, we have to use approximate methods. For example, in [7-9] there was used the approximation of electron tunneling from a potential well with an attractive $\delta$-potential. In this case such approximation is adequate, because the surface electronic state released after tunneling was almost instantly filled by another electron. As a result, the calculated CVCs of the tunneling current in low electric fields were compiled to form: $I \sim U^{(4-n) / 2}$ (the index $n=0,1,2$ is determined by the dimension of the initial electronic state). In the particular case of free lateral surface electronic states (such as Tamm levels) $n=2$ the result being to the standard $[6,10]$.

The traditional theory of tunneling current in STM [6] did not take into account the influence of the structure of the initial surface electronic states on the CVC. The reason is that the tunneling probability in an electric field is estimated rather crudely, in a purely exponential approximation, in fact repeating for a long time Fowler and Nordheim approximation. However, exactly the wave functions of the surface electrons included in the matrix elements of the tunnel junctions determine the structure of the pre-exponential factors in the tunneling current formula [11]. These factors can be identified by making the appropriate tunnel CVC normalization. It turns out that such a "normalized" CVC has the simple form of a power function, and the exponent is the number of degrees of freedom of the surface electron. Since surface electrons localize on structural defects, the degree of local CVC deviation from linearity (deviation of $n=$ 2) can serve to a certain degree as an indicator of the quality of the local surface structure. In fact, a set of surface electronic states determines the fractal structure of the surface. This has, in one way or another, impact on all surface and emission properties of the body.

\section{Theory}

As mentioned in the introduction, the exact solution of the electron tunneling from the real surface of the semiconductor is almost impossible. However, this process relates to a broad class of phenomena induced by an external electric field. Consistent calculation of the tunneling current in STM with the pre-exponential factor in the tunneling probability is given in [11]. Here we briefly describe only the main points of this calculation, based on analogy with other areas of physics. Similar phenomena have been studied in almost all sections of quantum physics - nuclear physics, solid state physics, quantum field theory, etc. [8, 12-13]. Despite many differences, all such processes have a number of common features. The most important feature, for example, is a possibility to express the cross section and the probability of the relevant processes in terms of bilinear combinations of Airy functions. The argument of these functions is the ratio of twocharacteristic length parameter: $u= \pm \lambda / l$. The first parameter is similar to the length of de Broglie wavelength (in relativistic - Compton wavelength) and characterizes the electron mobility. For this situation, the order of $\lambda=\hbar(2 m W)^{-1 / 2}$, where $\hbar$ - Planck constant, $m$ - mass of the electron (in a semiconductor - effective mass), $W$ - height of the potential barrier between the tip and the sample (Fig. 1). The second parameter - the distance at which the electric field does work specific for this process $l=W / e E$, where $e$ - electron charge, $E$ electric field. Positive values of the parameter $u$ correspond to the energy range in which such process may occur without an external field. External electric field causes oscillations of the cross sections and probabilities due to the interference of de Broglie waves incident and reflected from the barrier created by the electric field [9].
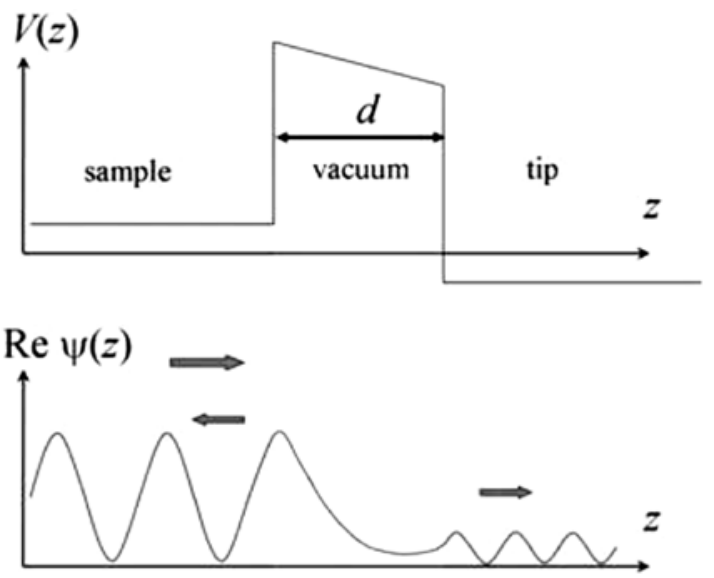

Fig. 1. The trapezoidal potential barrier energy structure and the real part of the electron wave function near the boundary of the sample and the probe.

If $u<0$ in the absence of the field the energy of the particle for transmission process is not enough, so it is only the electric field that increases the energy of electrons (no matter real or virtual). In this case, either cross sections or probabilities decrease monotonically with increasing energy deficit. If also $|u|<<1$ the probability is suppressed exponentially and the process has the tunneling nature. We are considering the field emission at low voltages that is precisely this type. Only in electric field (this corresponds to the length $l$ of the triangular potential barrier, i.e., the potential barrier on the right side in Figure 1 is the probe potential) the tunneling probability $p$ can be written in a universal form, where $D$ - is a constant that does not depend on the external field $[7,11]$

$$
p=D u^{(2-n) / 2} \exp (-4 / 3 u)
$$

Above-described scheme is also consistent with quantum field processes: photo-generation of electron-positron pairs, nuclear processes, induced by beta decay and the splitting of the deuteron, internal photoelectric effect in semiconductors (Franz-Keldysh effect), auto-ionizing atoms and a number of similar threshold processes, 
induced by an electric field. The main issue for us here is the factors that determine the $n$ in eq. (1). We can simplistically assume that $n$ is determined mainly by the symmetry of the initial state. In this respect, the process of field emission from a semiconductor to a metal probe is rather typical. If electron tunneling came from (zero-dimensional) $s$-state to a free state with a continuous spectrum, $n=0$ (the same as potential of $s$ electron ionization from delta-quantum well).

Due to the fact, that the transition goes to the final state on the metal probe the exponent increases to two. Indeed, the electron transfer is possible only into the free state with zero Fermi function, and the number of such states, as noted above, is proportional to the external field. Since $u \sim E \sim U$ then the contribution of zero-dimensional surface states in the CVC of the tunnelling current depends on the external field as $I \sim U^{2}$. This is fully consistent with the wellknown classical result of Fowler and Nordheim. In onedimensional states occurring in linear structural defects (grain boundaries, edges of the steps, etc.); there is an additional degree of freedom. This gives an additional integral over the momentum variable in the calculation of the contribution to the current. So it enables calculating the contribution to a current over the momentum variable. This integral reduces the degree of $u$ in the tunnelling probability and $U$ respectively by $1 / 2$, when determining the contribution to the tunnelling current. Respectively, in the lateral delocalized states with two degrees of freedom there would be two integrals and the degree of $U$ is reduced to one: $I$ $\sim U[6,10,11]$.

In analytical form, the density of the tunneling current from the surface of the semiconductor can be written as [10]

$$
j=e \int_{0}^{W_{m}} p\left(W_{z}\right) \xi\left(W_{z}, e U\right) d W_{z}
$$

where $p\left(W_{z}\right)$ - the probability of electron tunneling, and the integration should be made over the electron energy $W_{z}$ along the axis normal to the surface; $W_{m}$ - its maximum value. Value $\xi\left(W_{z}, e U\right)$ being usually referred to as the density of surface electronic states. However, the dimension of this magnitude (SI $\mathrm{m}^{-}$ $2 * c^{-1} * J^{-1}$ ) shows that it is the flux density of particles per unit energy interval. It is proportional to the density of occupied states in the semiconductor and the density of free states in the metal (the probe). After applying the voltage to the probe, the electron states close to the Fermi level in the metal are "liberated". The result is the current from the surface of the semiconductor to the metal. Probability $p\left(W_{z}\right)$ has the form eq.(1) only for the triangular barrier in the absence of the other fields except the electric field. In this case, the barrier for electron tunnelling extends the entire length $l$. For a thin trapezoidal barrier (length $d \ll l$ ) sub-barrier electron trajectory is much shorter (Fig. 1). Calculating the tunneling exponent in the usual manner [11], we obtain:

$$
\begin{aligned}
& -2 \int_{0}^{d} d z\left(W_{0}-e E z\right)^{\frac{1}{2}}= \\
= & -\frac{4}{3 e E}\left[W_{0}{ }^{3 / 2}-\left(W_{0}-e E d\right)^{3 / 2}\right] \approx \\
\approx & -2 W_{0}{ }^{1 / 2}-e E d^{2} / 2 W_{0}{ }^{1 / 2}
\end{aligned}
$$

where $W_{0}$ - the height of the barrier on the left side. In low fields, the second term is usually neglected, so that the field strength in the tunnelling exponent reduces. As a result, if we use the approximation of $n$ dimensional binding $\delta$-potential for low external fields $e U \ll W_{0}$, the tunneling probability of an electron with binding energy of $W=W_{0}+W_{m}-W_{z}$ (the distance of electron - vacuum levels on the energy scale) from the semiconductor surface can be represented as

$$
p \propto\left[\frac{e U}{d W_{0}^{3 / 2}}\right]^{(2-n) / 2} \exp \left(-H d W_{0}^{\frac{1}{2}}\right)
$$

where $H$ - a dimensional constant is not of interest to us. Function $\xi\left(W_{z}, e U\right)$, appearing in eq.(2), can be estimated as

$$
\xi\left(W_{z}, e U\right) \propto e U\left(W_{m}-W_{z}\right)^{\beta-1} .
$$

In fact, the number of states in the probe "liberated" by applying voltage is proportional to $e U$. The constant $\beta$ characterizes uniform filling of allowed states in areas such as the probe material and the studied surface. For example, for quasicontinuously filled conduction band $\beta=1$, for solitary or discharged surface electronic states $\beta » 1$, etc. Substituting eq. (3) - (5) in eq. (2), we obtain for the current density

$$
\begin{gathered}
j \propto \frac{e U^{(4-n) / 2}}{d^{(2-n) / 2}} \exp \left(-H d W_{0}^{\frac{1}{2}}\right) \cdot \int_{0}^{W_{m}} d W_{z} \\
\left(W_{m}-W_{z}\right)^{\beta-1}\left(W_{0}+W_{m}-W_{z}\right)^{-3(2-n) / 4} . \\
\exp \left[-H d\left(W_{m}-W_{z}\right) / 2 W_{0}^{\frac{1}{2}}\right]
\end{gathered}
$$

This is the Laplace integral, which can easily be estimated [11]. The main contribution to the integral comes from the region near the upper limit. As a result, one can get a final expression for the tunneling current to probe at low voltages $e U \ll W_{0}$

$$
\begin{aligned}
I(U) \approx & K U^{(4-n) / 2} \cdot W_{0}^{\beta / 2-3(2-n) / 4} \\
& \cdot d^{(n-2) / 2-\beta} \exp \left(-H d W_{0}^{1 / 2}\right)
\end{aligned}
$$

In special case $n=2, \beta=1$ this expression reduces to the standard formula [10]. It is not casually, since it is well known "ohmic" Simmons result obtained for the "metal-to-metal" tunneling by integrating over all possible values of the lateral moment. To get rid of extra constants normalize eq. (7) by an arbitrary data point $\left(I_{0}\right.$ ,$\left.U_{0}\right)$

$$
I / I_{0}=\left(U / U_{0}\right)^{(4-n) / 2}
$$


Sum up briefly the theoretical part of the work. The contribution of surface electronic states of various dimensions (symmetry) gives as a result the following asymptotic for the local CVC for small $U$, where the constants $A, B$ and $C$ give an idea of the contribution to the tunneling current: two-dimensional, one-dimensional and zero-dimensional states, respectively.

$$
I=A U+B U^{3 / 2}+C U^{2}
$$

Clearly, when $U \rightarrow 0$, the contribution of the second and the third term tends to zero, what explains the "vitality" of the traditional approach.

\section{Experiment}

It is well known that the electronic structure properties of bulk materials are influenced by crystallographic defects of different origin. Understanding of the degree of their influence on the electronic structure properties of poly- or mono-crystalline materials, such as carbonbased materials, is a necessary requirement if these materials are to be used for possible future electronic devices.

In a number of recent studies it was established the formation of three main types of structural defects on the surface of semiconductor materials based on carbon point, flat and volumetric [14-15]. The other structural defects such as linear quantum wires or extended nanometer height steps were less common.

All of them formed quasi-localized electronic states with the energy near the Fermi surface. Since the density of electronic states on the surface of intrinsic semiconductor near the Fermi level is usually quite low, the "added" electronic states of defects significantly affected the local electronic structure of the semiconductor surface and therefore the tunneling current to and from the surface. For example, usually on the graphite surface there are many extended steps of different heights similar one-dimensional quantum wires. In this case, it was recommended to measure the electronic structure of graphite no closer than 5-20 nm (depending on temperature) from the edges of the steps [16-17]. These distances depend on temperature: the higher the temperature, the shorter the distance. Monocrystalline graphite due to the peculiarities of its crystal structure and the possibility to obtain sufficiently simply an atomically clean surface is a model material for the study of the influence of structural defects on the electronic properties of the surface. Therefore, it makes sense to explore the more CVCs of the tunneling current from the graphite surface at different distances from the edge of the extended steps.

The highly oriented pyrolytic graphite (HOPG) ZYH type with misorientation angles (mosaic range) $3,5^{\circ}-5^{\circ}$ (NT-MDT, Russia) was used as a test sample. Clean surface of HOPG was obtained by cleaving with the adhesive tape. Then the sample was placed quickly in a high vacuum scanning tunneling microscope (STM Solver HV-MFM, NT-MDT, Russia), evacuated to a pressure of not more than $10^{-6} \mathrm{~Pa}$. Before STM measuring the sample was annealed in a high vacuum at $523 \mathrm{~K}$ to remove any surface contaminants, primarily water. As received the HOPG sample, like any other material, is not produced defect-free. Although the density of structural defects on flat atomic terraces is rather low, the existence of steps of different heights on the HOPG surface substantially increases their number. It is not always possible to observe such structural defects in the STM images even with atomic resolution. So getting independent information on the nature of structural defects and their location by using other methods is of practical importance.

When scanning and imaging the surface, the tunnelling parameters $I$ and $U$ determine the tip-tosubstrate distance $(d)$, the exact value of which is not well known. For the scanning tunneling spectroscopy (STS) the STM tip is moved to the position of interest with the feedback still activated. Therefore, $d$ is adjusted according to the selected bias voltage and the set point for the tunnel current, before the feedback loop was interrupted. The vertical and lateral position of the tip is kept fixed, whereas a bias ramp changes from -1.5 to + 1.5. The measurement of LTCVCs conducted in the voltage range of $\pm 0,5 \mathrm{~V}$, which was about an order less than the work function of graphite $(4.3 \mathrm{eV})$. At these voltages, the electric field can still be considered rather low, compared to the internal field. During the CVCs data collecting the tip-to-substrate distance and the selected tunneling current set point of $0.5 \mathrm{nA}$ were fixed according to the sample bias of $-1.5 \mathrm{~V}$ and. Since all CVCs were measured for a single scan, we could assume that in the process of measuring $d$ was not changed. At each selected point of the surface 10-15 CVCs were measured, the results were averaged and analyzed.

Unfortunately, the absolute value of $d$ is not accessible experimentally and remains unknown. The tunneling current is contributed due to emission from the surface electronic states of the region, the exact size of which is difficult to assess. These states include also the states, localized on different kinds of structural surface defects. Since the graphite samples are misoriented with respect to basal plane on HOPG, the formation of steps and flat terraces on the clean surface is inevitable and well seen in Fig. 2.

Two series of measuring were conducted. The first consisted of four CVCs, taken on the different points along the edge of the step (Fig.2a), and the second - of five CVC measured at flat terrace at different distances from the step edges (Fig. 2b). 

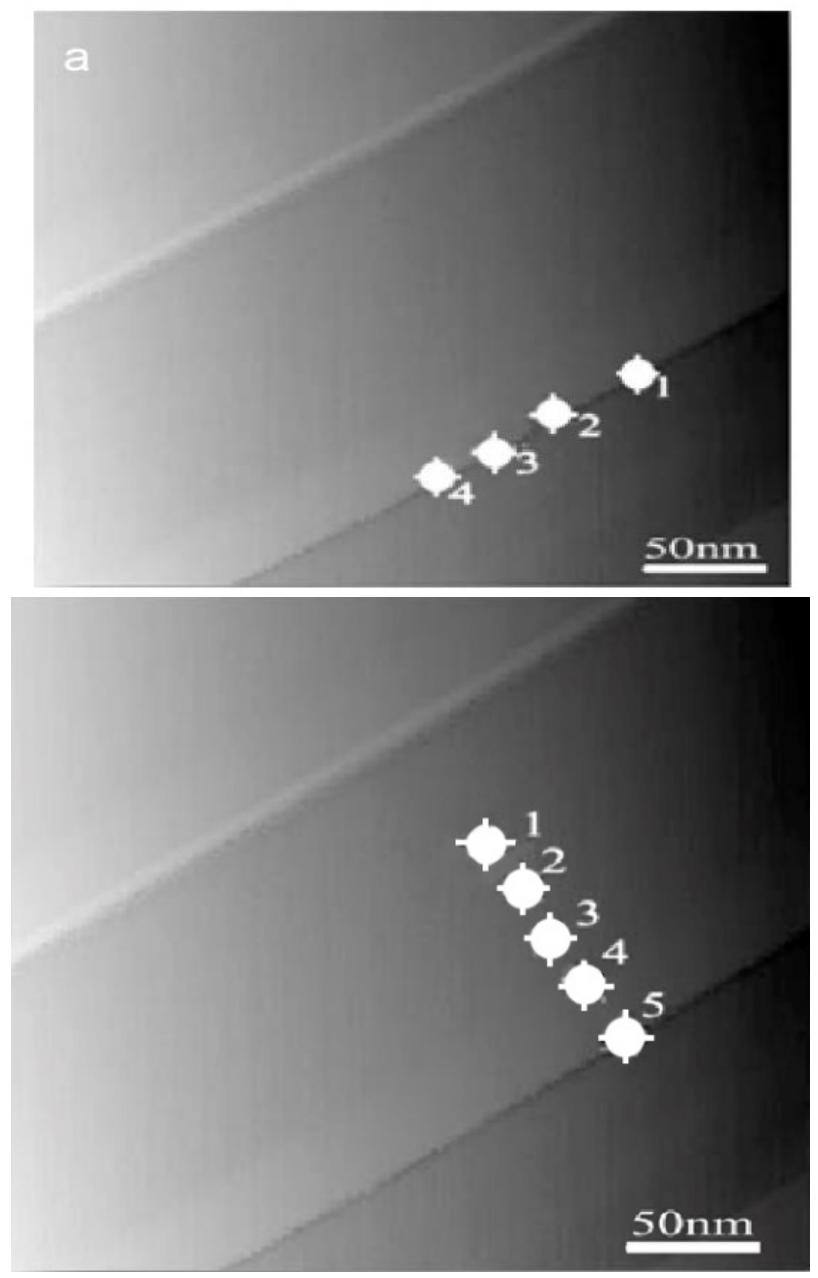

Figure 2. Points on the surface of graphite, in which the LTCVCs were measured: along the edge of the step (a) and perpendicular to the edge of the step (b) (point 5 is common).

These distances are given in Table 1.

Table 1. Position of the points of CVC measured on a flat terrace

\begin{tabular}{|c|c|c|}
\hline $\begin{array}{l}\text { The point at } \\
\text { which the CVC } \\
\text { is measured on } \\
\text { the terrace }\end{array}$ & $\begin{array}{l}\text { The distances } \\
\text { along the step } \\
\text { from the point } 5 \text {, } \\
\mathrm{nm}\end{array}$ & $\begin{array}{l}\text { The distance } \\
\text { from the edge } \\
\text { of the upper } \\
\text { step, nm }\end{array}$ \\
\hline 1 & 94 & 52 \\
\hline 2 & 72 & 74 \\
\hline 3 & 48 & 97 \\
\hline 4 & 26 & 117 \\
\hline 5 & 0 & 144 \\
\hline
\end{tabular}

Corresponding local tunnel CVCs and their corresponding dependencies of differential conductance versus applied voltage are presented in Fig. 3a and 3b.
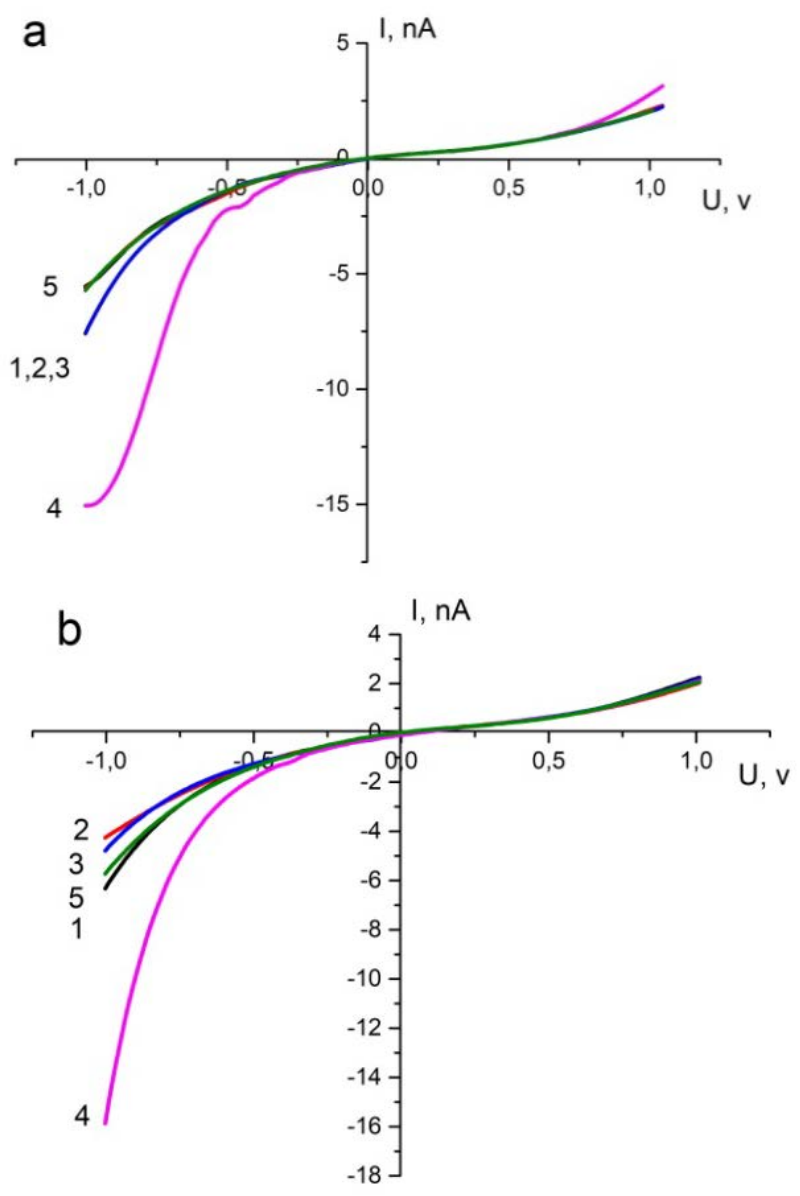

Figure 3. LTCVCs, measured at points along the edge of the step (a) and along the normal to the edge of the step (b).

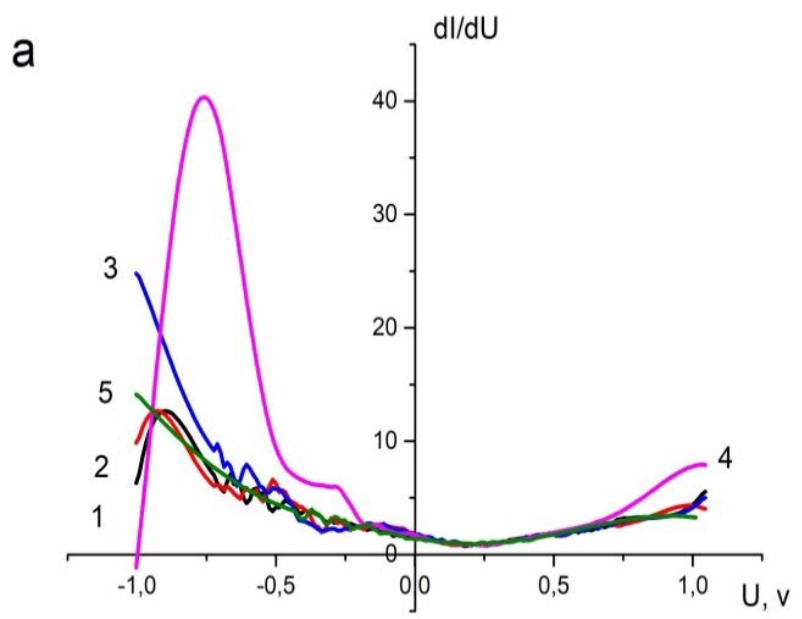




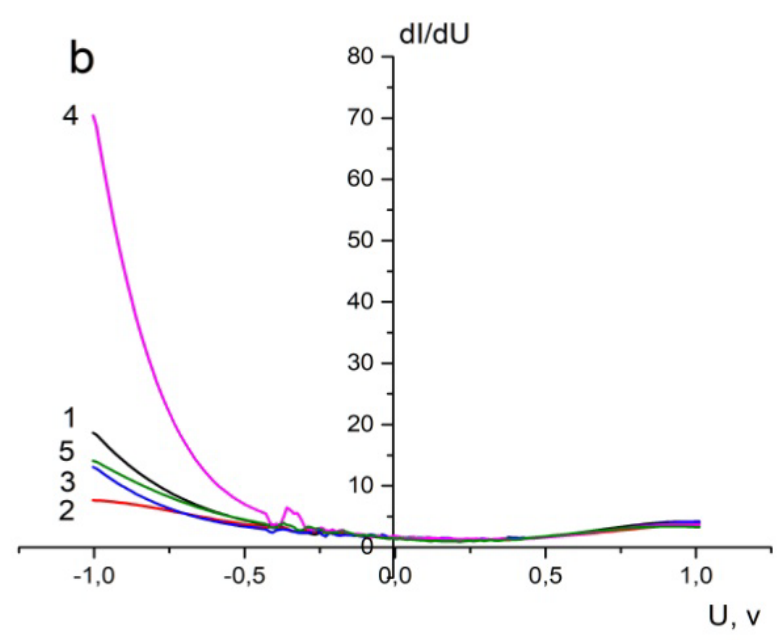

Figure 4. Differential conductance versus applied voltage, measured at points along the edge of the step (a) and along the normal to the edge of the step (b).

The results of mathematical processing of CVCs are shown in Tables 2 and 3.

Table 2. The coefficients of the approximation curve for the points along the edge of the step. Sign of the branches corresponds to the sign of the probe potential

\begin{tabular}{|r|c|c|c|c|}
\hline Point & Branch & A & B & C \\
\hline 1 & + & 0,2895 & 0,3158 & 0,3947 \\
\hline 1 & - & 0,9821 & 0,0060 & 0,0120 \\
\hline 2 & + & 0,3241 & 0,2990 & 0,3769 \\
\hline 2 & - & 0,9743 & 0,0069 & 0,0294 \\
\hline 3 & + & 0,4984 & 0,3271 & 0,1745 \\
\hline 3 & - & 0,9791 & 0,0004 & 0,0246 \\
\hline 4 & + & 0,1216 & 0,0946 & 0,7838 \\
\hline 4 & - & 0,9923 & 0,0010 & 0,0069 \\
\hline
\end{tabular}

Table 3. The coefficients of the approximation curve for points across the step surface. Sign of the branches corresponds to the sign of the probe potential

\begin{tabular}{|c|c|c|c|c|}
\hline Point & Branch & A & B & C \\
\hline 1 & + & 0,5650 & 0,0003 & 0,4346 \\
\hline 1 & - & 0,8730 & 0,0397 & 0,0873 \\
\hline 2 & + & 0,5224 & 0,0003 & 0,4773 \\
\hline 2 & - & 0,8196 & 0,0074 & 0,1730 \\
\hline 3 & + & 0,7127 & 0,0034 & 0,2840 \\
\hline 3 & - & 0,8561 & 0,0227 & 0,1212 \\
\hline 4 & + & 0,2937 & 0,0093 & 0,6970 \\
\hline 4 & - & 0,8769 & 0,0077 & 0,1154 \\
\hline 5 & + & 0,3069 & 0,2354 & 0,4577 \\
\hline 5 & - & 0,9789 & 0,0017 & 0,0231 \\
\hline
\end{tabular}

Each CVC was approximated by eq. (9) for the forward and backward branches, and optimal values of the coefficients were selected by the standard method of least squares.

\section{Discussion and conclusion}

Let us first consider the data in Fig. 4, which shows the distribution of the local density of electronic states (LDOS) as a function of energy. It is seen that, depending on the point chosen for measurements on the graphite surface, LDOS can vary significantly. In this case, the edge of the valence band (a sharp change of LDOS in the $\mathrm{E}<\mathrm{E}_{\mathrm{F}}$ region) manifests itself in most of the spectra obtained. At $E>E_{F}$ in the region of free (vacant) states, the dependence of LDOS on energy in measurements along the edge of the step (Fig. 4a) has a different form compared to practically indistinguishable curves in measurements along the normal to the edge of the step (Fig. 4b). In the region of negative and positive voltages, oscillations of the tunneling current are observed, which have a regular character, in no way associated with noise during measurements and are not eliminated by smoothing. The strongest oscillations are observed in the range of negative voltages. According to [18-20], such oscillations can phenomenologicaly be attributed to localized $\pi$-states.

Turn first to the LTCVCs measurements at the points along the edge of the step. According to [16], in this case, one should expect a significant contribution to the tunneling current the one-dimensional states. The results of such treatment are presented in Table 2. As can be seen from the data of Table 2, there is a striking difference of forward (the flow of electrons from the sample to the probe) and the reverse CVC branches. Reverse branch - almost straight ohmic curve (factor A is very close to unity, and $B$ and $C$ are negligible). At the same time the forward branch has a significant curvature (all coefficients are comparable). This is consistent with the above model, as the shape of the CVC curve is determined basically by the initial symmetry of the electronic states (at the sample in the case of forward branch and the probe in the case of the reverse branch). On a graphite surface electrons are transferred from the states of various dimensions, which results in noticeable values of the coefficients $B$ and $C$, while the surface state of the metal probe, as shown in Table 2, are not localized inside the metal.

This is consistently well with the conclusions of [13], where the theoretical model was proposed for the metalmetal tunneling (about the nature of differences in the forward and the reverse branches of CVC see also [7]). At points 1-3, the contribution of all three types of states is comparable. The point 4 on the forward branch markedly knocks out of this series, which may indicate the local concentration of defects in its vicinity. For example, this may be defects on the step edge such as "zigzag" or "chair" type, described in [17]. The differences of the CVC forward branch in point 4 of the other points can be observed visually from Fig. 4. To confirm our model it is important that on the reverse branch of CVC there is practically no effect at point 4 .

Consider the next series of measurements on the flat surface of the terrace. The summation of approximation coefficients in Table 3 shows that the coefficients for point 5 along the line perpendicular to edge of the step on the forward branch as well as on the reverse branch of the CVC are quite consistent with similar coefficients for points 1-3 of the previous series. In other words, point 5 is quite a typical point on the edge of the step.

Secondly, in the CVCs, measured in 1-4 points, the coefficient B sharply decreases (almost to zero), i.e. the contribution of one-dimensional initial states decreases. 
It is also quite understandable, because the abovementioned states are localized on the linear defects. As mentioned before, the reverse branch of the CVC is much closer to a straight ohmic line than the forward branch. In this series of measurements deviations from Ohm's law (or more precisely, the values of the $\mathrm{C}$ coefficients) are more significant than in the previous ones. Concerning the forward CVC branches of 1-4 points on a flat terrace surface, its substantial nonlinearity may be associated not only with it, but with the existence of point surface defects, which are difficult to visualize. However, in general, the experimental data are consistent with the proposed model.

Of course, it would be possible to draw a conclusion about one correspondence of $\mathrm{B}$ and $\mathrm{C}$ coefficients to the local density of surface defects corresponding symmetry only after comparing them with the results of defect measurements by independent methods.

Nevertheless, these results give a basis for possible practical applications of the obtained parameters. First, if the measured degree of localisation of the surface electron is significantly greater than 1 , then the presence of defects on the surface structure should be supposed. These hidden defects cannot always be visualized even with probe microscopy.

Secondly, in the STM constant tunneling current mode the topography of the studied surface depends not only on $d$, but also on the electron density distribution on the surface of the sample. Therefore, the situations may exist, when some corrections of the measured topography should be made. Of course, the value of $n$ is not the only factor that determines a deviation of the observed CVC from a linear law or other simple dependences. However, if other factors are excluded, the value of $n$ should be taken into account as a parameter treating a topology of surface defects.

\section{Acknowledgements}

This study was supported by the Ministry of Higher Education and Science of the Russian Federation grants NFSFS-2020-0025 and carried out using equipment provided by the Center of Collective Use of MSUT "STANKIN".

\section{List of symbols}

$I$ - tunneling current

$U$ - voltage at the probe

$n$ - dimension of the initial electronic state on the surface

$u$ - universal parameter characterizing the influence of an external electric field on a process

$\lambda$ - de Broglie wave (or Compton) of the tunneling electron

$l$ - electrical length or distance at which the electric field does the work required for process execution (in pure electric field coincides with the length of the triangular potential barrier).

$\hbar$ - Planck constant $m$ - mass of the electron (in a semiconductor - effective mass)

$W$ - electron binding energy (for tunneling from the surface is equal to the local work function).

$j$ - tunneling current density of (from) the semiconductor surface.

$e$ - elementary charge.

$E$-electric field

$p$ - quantum mechanical probability of the process (eg, tunneling from a potential well at the semiconductor surface).

$D, H$ - constants, containing the studied variables

$W_{z}$ - kinetic energy of the electron motion along the axis $\mathbf{z}$, normal to the surface.

$W_{m}$ - maximum value of the kinetic energy of the electron along the axis z.

$\xi\left(W_{z}, e U\right)$ - so-called energy density of surface electronic states, to be exact - the electron flux density of the energy surface

$d$ - distance between the probe and the surface, or the width of the trapezoidal potential barrier

$\beta$-parameter of degree of uniformity of filling surface electronic states.

\section{References}

1. M. Celestin, S. Krishnan, Sh. Bhansali, E. Stefanakos, Nano Research, 7(5), 589 (2014)

2. A.I. Loskutov, O.Ya. Uryupina, S.N. Grigoriev, V.B. Oshurko, V.I. Roldughin, Colloid Journal, 75(3), 301 (2013)

3. H. Luth, Solid Surfaces, Interfaces and Thin Films.(Springer, Berlin, 2010)

4. M. Grundmann, The Physics of Semiconductors (Springer, Berlin, 2006)

5. J. Martin, N. Acerman, G.Ulbricht, T.Lohmann, J.H. Smet, K. von Klitzing, A. Yacoby, Nat. Phys., 4(144),. 46-89 (2008).

6. J. Tersoff, D. Hamann, Phys. Rev. B, 31(2), 805 (1985).

7. V.N. Rodionov, G.A. Kravtsova, A.M. Mandel, JETP Letters, 75(4), 363 (2002)

8. V.G. Kadyshevskii, G.A Kravtsova, A.M. Mandel,V.N. Rodionov, Theoretical and Mathematical Physics, 134(2), 198 (2003)

9. V.N. Rodionov, A.M. Mandel, E.V. Arbuzova, arXiv: hep-ph/0510357v1, 11 (2005)

10. J. G.Simmons, J. Appl. Phys., 34, 1793 (1963)

11. M. Mandel, S.N. Grigoryev, A.I. Loskutov, V.B. Ohurko, G.I. Solomakho, Journal of Computational and Theoretical Nanoscience, 12(11), 1 ( 2015)

12. N.L. Manakov, M.V. Frolov, A.F. Starace, I.I. Fabrikant, J. Phys. B: At. Mol. Opt. Phys., 33, R141 (2000)

13. V.N. Rodionov, G.A. Kravtsova, A.M. Mandel, JETP Letters, 78(4), 218 (2003)

14. A.A. Kelly, K.M. Knowls, Crystallography and Crystal Defects (Willey\&sons, London, 2012)

15. D.D.L. Chung, J. of Mater. Sci., 37, 1475 (2002) 
16. M. Fujita, K. Wakabayashi, K. Nakada, K. Kusakabe, J. Phys. Soc. Jpn, 65, 1920 (1996)

17. Y. Niimi, T. Matsui, H. Kambara, K. Tagami, M. Tsukada, H. Fukuyama, Phys. Rev. B, 73, 085421 (2006)

18. R.M. Feenstra, J.A. Stroscio, A.P. Fein. Phys. Rev. Lett., 58, 1192 (1987)

19. R.M. Feenstra, J.A. Stroscio. J. Vac. Sci. Technol.B, 5(4), 923 (1987)

20. R.M. Feenstra, Phys. Rev. B, 50(7), 4561 (1994) 